\title{
Effects of oil palm tocotrienol rich fraction on the viability and morphology of astrocytes injured with glutamate
}

\begin{abstract}
Tocotrienol-rich fraction (TRF) is an extract of palm oil that consists of $25 \% \alpha$-tocopherol and $75 \%$ tocotrienols. TRF was shown to possess antioxidant, anti-inflammatory, anticancer, neuroprotective and cholesterol-lowering activities. Glutamate is the major mediator of excitatory signals in the mammalian central nervous system. Extreme amounts of glutamate in the extracellular spaces can lead to numerous neurodegenerative diseases. Hence, the efficacy of oil palm TRF and $\alpha$-tocopherol in protecting astrocytes against glutamate-induced cell death was studied. Specifically, the effectiveness of pre- or post-treatment of TRF and $\alpha-$ tocopherol upon glutamate excitotoxicity was determined by evaluating cell viability and morphology of astrocytes. Cell viability was measured using MTT assay while cell morphology was monitored under fluorescent microscope using the acridine orange/propidium iodide (AO/PI) assay. Exposure to $230 \mathrm{mM}$ glutamate significantly reduced cell viability to $50 \%$ in both the pre- and post-treatment studies; however, pre- and post-treatment with TRF and $\alpha$-tocopherol attenuated the cytotoxic effect of glutamate. Compared to glutamate-injured astrocytes, pre-treatment with 100, 200 and $300 \mathrm{ng} / \mathrm{ml} \mathrm{TRF}$ significantly improved cell viability following glutamate injury to $86.6 \%, 86.7 \%$ and $93.9 \%$, respectively $(\mathrm{p}<0.05)$. On the contrary, high concentrations of $\alpha$-tocopherol promote cell death. This study shows that TRF not only provide a better protection against glutamate toxicity (pre-treatment), but was also able to reverse the lipid peroxidation resulting from glutamate insults (post-treatment). The present results demonstrate that TRF, but not $\alpha$ tocopherol, protected the astrocytes against glutamate-induced cell death, indicating its neuro-protective potential.
\end{abstract}

Keyword: Cryoconcentration; Centrifugation; Blueberry juice; Solutes; Polyphenol monomers; Process parameters 